\title{
Reflexiones desde el aula. Acerca DE LA CONFIGURACIÓN HISTÓRICA Y EPISTEMOLÓGICA DEL OBJETO DE INTERVENCIÓN EN TRABAJO SOCIAL ${ }^{1}$
}

REFLECTIONS FROM THE CLASSROOM ABOUT THE HISTORICAL

*Estudiante de cuarto semestre de Trabajo Social, Universidad de Cartagena. Miembro del semillero del Grupo de Investigación Cultura ciudadanía y poder en contextos locales.

**Estudiante de cuarto semestre de Trabajo Social, Universidad de Cartagena. Miembro del semillero del Grupo de Investigación Cultura, ciudadanía y poder en contextos locales

***Estudiante de cuarto semestre de Trabajo Social, Universidad de Cartagena. Miembro del semillero del Grupo de Investigación Cultura, ciudadanía y poder en contextos locales.

****Estudiante de cuarto semestre de Trabajo Social Universidad de Cartagena. Miembro del semillero del Grupo de Investigación Cultura, ciudadanía y poder en contextos locales.

*****Estudiante de cuarto semestre de Trabajo Social, Universidad de Cartagena. Miembro del semillero del Grupo de Investigación Cultura, ciudadanía y poder en contextos locales.

1 Este ensayo de reflexión, es resultado de un ejercicio académico de la Asignatura de Epistemología de Trabajo social, coordinado por el profesor Alexander Pérez Álvarez. Las autoras se encuentran en tercer semestre de su formación y son integrantes activas de semillero del grupo de investigación Cultura, ciudadanía y poder en contextos locales.
AND EPISTEMOLOGICAL CONFIGURATION OF THE PURPOSE OF

INTERVENTION IN SOCIAL WORK

Por: Juliana Amaya Paulo*

Carmen Melissa González Flórez ${ }^{* *}$

Estefani Gulloso Hernández ${ }^{* * *}$

Angélica María Ramírez Martelo ${ }^{* * * *}$

Natalia Paola Torres López ${ }^{* * * * *}$

\begin{abstract}
"Trabajo Social no es algo inmutable, es una construcción
Social que toma forma desde una teoría y metodología

que dirige la acción del profesionista."

Nelia Tello Peón, Trabajadora Social
\end{abstract}

\section{RESUMEN}

Este artículo es un ensayo producto de un estudio documental realizado en el marco de la asignatura Epistemología del trabajo Social. En este ejercicio se presenta de manera sistemática una reflexión epistemológica frente a las vertientes teóricas que han permitido dotar de sentido el objeto de intervención en Trabajo Social. Desde una perspectiva histórica, se retoman aportes realizados por diversos autores y autoras en Iberoamérica sobre las diferentes conceptualizaciones teóricas que se han realizado alrededor del Objeto de intervención del Trabajo Social, como una categoría clave de la disciplina que se ha constituido de manera dialéctica y por tanto en constante tensión y debate al momento de la delimitación teórica del mismo.

Palabras clave: objeto de intervención, intervención social, trabajo social, re-conceptualización.

\begin{abstract}
This article is an essay product of a documentary study carried out within the Epistemology of Social Work subject. In this exercise a systematic epistemological reflection is presented taking into account the theoretical aspects that have allowed the purpose of intervention in Social Work makes sense. From a historical perspective, contributions made by various authors in IberoAmerica are taken up on the different theoretical conceptualizations that have been carried out around the Purpose of Social Work Intervention as a key category of the discipline that has been
\end{abstract}


Juliana Amaya Paulo, Carmen Melissa González Flórez, Estefani Gulloso Hernández, Angélica María Ramírez Martelo, Natalia Paola Torres López

constituted in a dialectical way and by both in constant tension and debate at the time of the theoretical delimitation of it.

Keywords: purpose of intervention, social intervention, social work, re-conceptualization.

\section{Introducción}

nalizar el objeto de intervención del Trabajo Social implica la
realización de un barrido histórico y analítico de los aportes que
brindan diversos autores y autoras sobre esta categoría, construida por medio de un proceso dialectico generado por las múltiples miradas epistemológicas que han sido sujetas a debates, que han dado paso a una parte de la consolidación teórica del Trabajo Social como una disciplina de las Ciencias Sociales.

En la construcción del presente análisis traeremos a colación dos categorías fundamentales que surgen como prerrequisito para poder abarcar las diferentes conceptualizaciones que se han realizado entorno al objeto de intervención, como lo son el Trabajo Social y la intervención social.

Las definiciones sobre el objeto de intervención del Trabajo Social y sus determinados aportes han sido en algunos casos divergentes y poco estáticas; estas a su vez varían de acuerdo a la perspectiva teórica en la cual se sitúe el autor o la autora, así como también de acuerdo la época y lugar en la que han sido pensadas.

Como punto de partida situaremos nuestro análisis desde el Movimiento de Reconceptualización del Trabajo Social² ${ }^{2}$ que ocupó lugar en Latinoamérica donde surge el interés teórico y profesional del Trabajo Social, y también el interés por la definición y el establecimiento de un objeto de intervención dentro de la profesión.

Analizar las vertientes teóricas sobre las cuales se ha constituido el objeto de intervención en trabajo social, permite reconocer una profesión y disciplina que desde su naturaleza misma se interroga y está en una constante auto reflexividad. Estas preguntas por el objeto parecieran resultas en las últimas generaciones de trabajadores sociales, sin embargo no puede dejarse de lado que es una reflexión necesaria en una profesión y disciplina que se configura en las relaciones entre teorías, sujetos-actores y realidades particulares.

2 El movimiento de reconceptualización de trabajo social se da en América Latina a mediados de la década de 1960 en el siglo XX, con la intención de pensar y hacer un trabajo social cercano a la realidad social latinoamericana y romper con modelos funcionalistas importados de Europa y Estados Unidos. 


\section{Aproximaciones históricas de las categorías que anteceden al objeto de intervención.}

[ $]$

istóricamente se ha considerado y percibido al Trabajo Social como una labor altruista, caritativa, voluntarista y filantrópica, viéndose así como un conglomerado de diferentes componentes, teniendo como protagonista al ser humano y su papel en la sociedad, pensado desde una perspectiva estructural funcional, donde es necesario ubicar al individuo en alguno de los segmentos que la sociedad ha establecido.

Las sociedades han transitado por diferentes momentos históricos, entre ellos se destacan la trasformación de los sistemas de producción, pasando de tener un sistema netamente agrícola a uno industrial, brindando como resultado un cambio en las dinámicas sociales. Cabe resaltar que la necesidad integradora de la modernidad surge con la inserción del Estado-Nación como veedor del bienestar colectivo, por lo cual se sirvió del trabajo social y sus raíces filantrópicas para cumplir este fin. "El Trabajo Social nace como profesión con el desarrollo del estado de bienestar para atender lo social en las instituciones, apoyando a otros profesionistas" (Tello Peón, 2010, p.65).

En este sentido, Oscar Quintanilla (2013) considera que:

En la historia de la profesión de Trabajo Social la intervención ha transitado desde los fundamentos religiosos y humanistas, que sostienen las primeras prácticas voluntarias de asistencia y ayuda a los más necesitados, hasta las complejas relaciones de bienestar social que configuran la modernidad occidental a través del discurso del derecho y de los procesos socioeconómicos de la modernización.

La intervención del Trabajo social con relación a su historia, se ha permeado de las diversas lógicas y dinámicas en el contexto donde se ha visto inmersa la profesión situándose "en los problemas y conflictos sociales (...) para convertirse en un mandato sociojurídico cuya legitimidad reposó en el derecho, investidura que arropó al sujeto de la modernidad y lo situó como ciudadano titular de garantías y derechos". (Quintanilla, 2013, p.192).

Ahora bien, cuestionarse sobre la Intervención Social requiere establecer consideraciones sobre las primeras aproximaciones de la misma, que corresponden a la época moderna, así como también en torno las nuevas apuestas al rededor a esta categoría. La intervención en Trabajo Social es un 
Juliana Amaya Paulo, Carmen Melissa González Flórez, Estefani Gulloso Hernández, Angélica María Ramírez Martelo, Natalia Paola Torres López

proceso dinámico que se construye cuando se establece una reflexión sobre la realidad social y desde esta se construye el objeto de intervención.

Silvia Galeana y Nelia Tello, (2010) plantean que la intervención social se da entre la relación, sujeto-contexto-problema, del cual este último construido como el objeto de intervención que es a su vez la unidad de análisis que da paso a una unidad de trabajo, es decir, la intervención social.

\section{Contexto Histórico del Desarrollo del Objeto de Intervención}

n Latinoamérica el interés teórico por el objeto de intervención del Trabajo Social surge durante el Movimiento de Reconceptualización, entre los años 1960 y 1970 bajo el contexto de la finalización de la segunda guerra mundial y la imposición del capitalismo norteamericano como sistema económico de la sociedad. Este movimiento nace con la necesidad específica de articular el Trabajo Social con la realidad latinoamericana, que en esa época vivía en medio de la desigualdad social y la corrupción, ocasionada en parte por el mal manejo de las dictaduras preponderantes en países como Brasil, Uruguay, Argentina Y Chile.

El Trabajo Social latinoamericano durante la década de los 60 se encontraba fuertemente influenciado por sus raíces foráneas, "ocasionando una apropiación a-crítica de los referentes teórico-metodológicos principalmente de la escuela estadounidense, y en algunos casos de la escuela europea" (Chillito y otros, 2011, p. 6), alejándose cada vez más de la implementación de metodologías adecuadas para solución de los problemas que ocurrían en los países latinoamericanos, a diferencia de Estados Unidos que tenía establecida unas políticas públicas y una serie de lineamientos que generaban un adecuado bienestar para la sociedad.

En este orden de ideas, Norberto Alayón y Ma Lorena Molina (2004) aseguran que:

Las y los trabajadores sociales comenzamos a identificar y reconocer el origen de la desigualdad social en las relaciones de dominación vigentes en la sociedad, cuestionando las propuestas de integración al medio de los "desadaptados" o "marginados", propias de aquel pensamiento "modernizador" y de las concepciones teóricas funcionalistas, propuestas éstas provenientes de la óptica de entender como justo y adecuado el modelo imperante. Y se impugnaron las tendencias más tradicionales, previas aún al propio desarrollismo, que asumían la desigualdad social como una suerte de hecho natural. 
3 Según Carlos Barba Solano (2011), El campo de la integración social empezó a desarrollarse a partir de las reflexiones teóricas de Emile Durkheim acerca de lo problemático que resultaba integrar a las sociedades modernas caracterizadas por una gran densidad social y moral que acompañaba a los proceso de individuación y se traducía en una notable pluralidad de intereses y valores (Durkheim, 2007).
Es de este manera como se comienza a identificar el malestar social de la época, que respecta a las injusticias y desigualdades, donde los y las asistentes sociales mediados por su función de integradores sociales ${ }^{3}$, brindaban ayuda y seguimiento al "menesteroso", a través de "programas de corte desarrollista, con el fin de que este individuo se adaptara y cumpliera su respectiva función dentro del sistema." (Chillito y otros, 2011, p.6).

La teoría funcionalista de la época, implementada en el Trabajo Social, se puso en cuestión debido a su propósito de "integración", que en absoluto aceptaba las diferencias y exclusividades de individuos o colectivos, generando exclusión y marginación hacia ciertos sectores de la sociedad. Como consecuencia de esto, los y las aspirantes al ejercicio de la profesión comenzaron a sentir un rechazo hacia esta, y en la academia la deserción fue notoria.

Estela Grassi (1994) afirma que:

Con el Movimiento de Reconceptualización los trabajadores sociales de esta corriente asumieron para sí - de la manera más activa a lo largo de su historia- la tarea de conceptualizar tanto el objeto de su intervención como su práctica. El marco general de la Reconceptualización estuvo dado por: a) una fuerte politización de la sociedad en general; b) el desarrollo de corrientes críticas en las ciencias sociales, fundamentalmente de inspiración marxista (...) y c) el establecimiento de una relación más estrecha de estas corrientes con la práctica política. Paradójicamente, estas circunstancias - de hecho, movilizadoras de los cambios en el interior de la profesión- al combinarse con aquella tradición de activismo, no dieron lugar a la consolidación de una corriente crítica teóricamente sólida dentro de la profesión. (Norberto Alayón y Ma Molina 2004, p. 4).

Bajo este marco de situaciones se desenvuelve la definición del objeto de intervención, cuando las y los trabajadores sociales influenciados por las teorías críticas que están compuestas "principalmente de variados análisis críticos de diversos aspectos de la vida social e intelectual" (George Ritzer, 1993:163).

Asimismo decidieron repensar su labor técnica como un ejercicio teóricoprofesional, y es en ese momento cuando:

Emerge como preocupación el investir de cientificidad al trabajo social, convirtiéndolo en una disciplina social que produzca una teoría propia. De 
este modo, irrumpieron corrientes Dialéctico-Críticas y Constructivistas, que caracterizaron al conocimiento como producto social y perfilaron una fuerte tendencia hacia una epistemologización que siente las bases para lograr una transformación social."(Casá, 2014, P.7).

Para llevar a cabo esta tarea de Reconceptualización, era importante articular la práctica con la teoría; por lo que pensar teóricamente el objeto de intervención en el Trabajo Social, era uno de los principales objetivos de los y las trabajadoras sociales.

De acuerdo con González Saibene (2014):

El [Problema] Objeto de Intervención, entonces, se erigió en el imaginario académico profesional como un espacio de construcción de conocimientos que permitiría acercar a los trabajadores/as sociales a ese anhelado objetivo de construcción de un cuerpo teórico sólido. Y con ello se alcanzaría el estatuto científico que hasta el momento no se poseía.

Por otro lado, es importante resaltar como la poca clarificación del objeto de intervención en el Trabajo Social, ha significado un obstáculo, al momento de la implementación metodológica en la intervención social, "los diferentes autores que han realizado análisis teóricos sobre la profesión han eludido el problema del objeto, lo cual se convierte en una limitación para avanzar en la discusión de la metodología". (Gustavo Parra, 2007, 2).

\section{Configuraciones Teóricas del Objeto de Intervención}

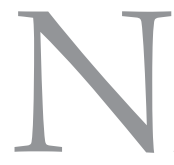

ridia Aylwin (1975) brinda un aporte pionero sobre el Objeto del Trabajo Social, teniendo "la posibilidad de definir el objeto del Trabajo

Social en términos de problemas sociales o problema social. La profesión es nacida justamente para enfrentar científicamente los problemas sociales, el Trabajo Social encuentra en ella su materia prima, su objeto de acción". (Martínez y Torrecilla, 2015: 233)

En el contexto en el que se sitúa este aporte, es posible identificar su influencia con los problemas sociales, que eran considerados como el objeto de accionar del Trabajo Social; el objetivo era hacer que el objeto- es decir el problemadesapareciera, dejando a un lado la autonomía total de los sujetos en función de los problemas que afectaban el orden social. 
Para Mercedes Escalda (1986), "el objeto es definido a partir de esta necesidad histórica y de su función social, señalando también que el objeto puede ser definido como los problemas sociales o como las metas a alcanzar, es decir, el bienestar social." (Gustavo Parra, 2007: 4).

De lo anterior es posible deducir que, aunque el objeto de intervención es percibido como los problemas sociales, la autora hace una nueva apuesta planteando que dichos problemas se encuentra mediados por necesidades que a su vez son históricas, es decir, cambian con los contextos. Además que en este periodo no era suficiente buscar solución a una situación, sino también con esta brindar un bienestar social.

Boris Lima (1989), influenciado por el pensamiento del Movimiento de Reconceptualización, afirma que:

Hasta ahora el objeto señalado por el Trabajo Social es el hombre desvalido, el menesteroso, el que entra en desequilibrio, desajuste o inadaptación con el orden establecido. Cualquier hombre que precise ser controlado y dirigido. Queremos desde ya avanzar que la materia $u$ objeto es aquello que una disciplina estudia y/o transforma por su acción y que según las particularidades del hacer del Trabajo Social (...) Se conforma más bien un sistema de objetos al considerar al hombre oprimido en su cotidianeidad frustrante. De allí que siempre existirá un objeto a investigar, a conocer, o sobre el cual se incursionará con arreglo a <métodos apropiados para que el propio objeto se convierta en sujeto de acción. Gustavo Parra (2007: 3).

De esta definición es posible interpretar que el autor se esfuerza por desarrollar una definición de la categoría objeto de intervención, pero a pesar de estar influenciado por una serie de teorías críticas, no es en ese sentido que la desarrolla, sino por el contrario se encuentra fuertemente arraigado al pensamiento positivista, previo al Movimiento de la Reconceptualización. En este sentido considera al objeto como el hombre desvalido y menesteroso, al que el trabajador social por medio de la implementación de métodos debe ayudarlo a convertirse a sí mismo en un sujeto de acción.

Ante la informalidad de la definición del objeto de intervención el centro latino americano de trabajo social CELATS en la década de los años 80 propone "definir el problema objeto de intervención es delimitar qué aspectos 
Juliana Amaya Paulo, Carmen Melissa González Flórez, Estefani Gulloso Hernández, Angélica María Ramírez Martelo, Natalia Paola Torres López

de una necesidad social son susceptibles de modificar con nuestra intervención profesional", el reconocimiento de las necesidades sociales es uno de los principales acercamientos al objeto de intervención, en este contexto, la lucha de clases sociales jugaba un papel fundamental para la transformación de la sociedad.

Margarita Rozas Pagaza (1990) por su parte afirma que el objeto de intervención "se construye desde la reproducción cotidiana de la vida social de los sujetos, explicitada a partir de múltiples necesidades que se expresan como demandas y carencias y que de esta forma llegan a las instituciones para ser canalizadas o no" (Martínez y Torrecilla, 2015, p.234).

Por otra parte, Estela Grassi (1995) considera que "el objeto es objeto de intervención, desde el momento en que su práctica está explícitamente dirigida a producir alguna modificación en la situación problemática puntual en relación a la cual es llamado a actuar." (Denise Casá, 2014, p.8) Es decir, que es en el campo de la intervención profesional donde se configura este objeto, y es considerado como tal -objeto de intervención- solo cuando él o la trabajadora social intervienen sobre el mismo, generando modificaciones en la problemática a la cual dicha intervención va a solucionar. En este sentido solo el objeto se constituye objeto cuando brinda una transformación mediante de su praxis.

En esta línea histórica, Alicia González (1995) afirma que "Ese objeto se construye en función de una problemática teórica que antecede a cualquier experiencia. Es el punto de vista teórico el que crea al objeto; éste es una construcción conceptual." (Denise Casá, 2014, p.8), asimismo plantea dos tipos de objeto que sintetizan lo propuesto en 1995 :

Un objeto empírico, se refiere a una situación particular caracterizada en y por su singularidad.

Objeto teórico, este hecho es lo que caracteriza a la producción científica, diferenciándose por ello de la intervención o ejercicio profesional, que persigue objetivos de modificación/transformación de aquella situación singular que es su objeto -empírico-. (González Saibene, 2014, p. 32).

Agregando que las construcción del objeto de intervención en el Trabajo Social se da mediante la articulación de los objetos teórico y empírico, bajo un campo de confrontaciones y tensiones. 
Maya de lozano (2008) afirma que:

El objeto de conocimiento e intervención, es lo social, lo que ha de entenderse como una dinámica compleja dentro de un sistema de interacciones que se dan en espacios eco-físicos, culturales y temporales particulares. (...) Lo social es caracterizado por los hechos institucionales, como producto del acuerdo entre los hombres, en un ámbito unitario en el que se dan problemas concretos y compartidos, de intercambios de mensajes culturales, sentimientos e intereses de bienes económicos. Es entonces la problemática social y los conflictos humanos generados en las relaciones dadas en la estructuras del sistema social, entendida esta como espacio temporal y cultural, la que precisa el objeto del trabajo social. (Rivera Alarcón, 2014: 2).

Desde una mirada del interaccionismo simbólico, donde los factores socioculturales y las interacciones entre las personas juegan un papel importante en la comprensión de los significados de las problemáticas sociales, es donde se precisa el objeto de intervención del trabajo social, comprendido por Maya de Lozano además como el objeto de conocimiento.

\section{Énfasis sobre la poca clarificación teórica del objeto de intervención}

Tr a conceptualización de la profesión ha transitado por un devenir historio y analítico desarrollado por autores como Kruse (1976), quien se J $\_$cuestiona el hecho sobre el ¿cómo es posible construir teoría del trabajo social si aun en la práctica de la misma no se ha tenido claridad de su accionar?

“(...). La no clarificación de la naturaleza del objeto repercute, entonces, sobre una de las patas del trípode en que se apoya la teoría. ¿Cómo queremos extraer saber de la práctica para enriquecer la teoría si un tercio de la teoría no tiene claro cuál es la práctica que le corresponde hacer?..." Kruse, Herman (1976) citado por Gustavo Parra (2007).

En Trabajo Social se han desarrollado una serie de debates con relación a su objeto de intervención, debido a su poca claridad y a su escasa participación teórica dentro de las demás disciplinas de las ciencias sociales. En relación a estos debates, se tiene la postura de Molina, Lorena y Romero, Cristina (1999), quienes planteaban que si se quería transitar del trabajo social como profesión a una disciplina científica, esta requería tener un objeto de intervención clarificado. 
Juliana Amaya Paulo, Carmen Melissa González Flórez, Estefani Gulloso Hernández, Angélica María Ramírez Martelo, Natalia Paola Torres López

En palabras de Alejandro Del Valle y María Sol Ramella (2009) la no definición a priori del objeto de intervención, no es más que el resultado de una realidad social variada, la cual por el hecho de ser cambiante se le es imposible predecir su objeto de intervención "antes de", obligándonos de esta manera a hacer construcciones es decir, delimitaciones conceptuales de los problemas cotidianos que se den en un contexto especifico, para de esta manera llevar a cabo su proceder profesional. También Silvia Galeana y Nelia Tello (2010) plantean que aquello que agudiza la imprecisión del conocimiento del quehacer profesional, es entre otras cosas: la complejidad de lo social, la intensiva práctica empírica y la falta de discurso. Es decir, la poca reflexión sobre la acción que se ejecuta en esta disciplina, es lo que en cierta manera impide la recreación de su conocimiento.

Nelia Tello (2008) plantea que:

Para comprender la situación problema objeto de nuestra intervención es necesario conocer el entorno social, sus narrativas, sus representaciones, sus códigos. Las relaciones sociales que significan su vida individual, grupal y colectiva, los modos en que los actores impregnaran de sentido su vida cotidiana y el mundo en el que viven. (Silvia Galeana, Nelia Tello, 2010: 29).

En este orden de ideas se pretende conocer la situación problema (objeto de intervención) mediante una perspectiva histórica hermenéutica, por lo cual se busca efectuar un proceso de interacción entre los sujetos sociales y el contexto donde estos se encuentra sumergidos, pues, es de esta manera según lo planteado por Tello Peón (2010) como él y la trabajadora social podrían llegar a intervenir.

\section{Consideraciones finales}

n este punto nos planteamos: ¿Es el objeto de intervención un sujeto, $\checkmark$ una necesidad o un problema social? La idea del objeto de intervención como una construcción, deviene a la aceptación sobre las diversas perspectivas que existen en torno a este, pertenecientes a teorías de las ciencias sociales. La construcción de un objeto proporciona a las y los Trabajadores Sociales la posibilidad de establecerlo de forma cambiante.

Después del Movimiento de Reconceptualización del Trabajo Social el cual buscaba la teorización y la profesionalización de la práctica y su quehacer; pese a las luchas que se han llevado a cabo aún se tiene el imaginario de que las 
y los trabajadores sociales intervienen solo desde la praxis, reduciendo a un segundo plano la investigación, la cual nos permite desarrollar la cientificidad del Trabajo Social, que a su vez da paso a la producción de conocimientos teóricos, por tal razón se debe propiciar desde la academia ambientes de debates que permitan la profundización y análisis de los mismos.

En esta línea podemos afirmar que el objeto de intervención no está dado "antes de" sino que por el contrario se construye en el mismo campo de la intervención, por lo cual observamos a profesionales con dificultades a la hora de identificar el problema del objeto de intervención; sin embargo, ello más que un obstáculo epistemológico u ontológico es una característica intrínseca a la profesión- disciplina, en el sentido que el objeto nunca puede ser a priori, sino producto de una construcción colectiva entre los diferentes actores-sujetos que intervienen e interactúan en un espacio, campo problemático o escenario social. Este encuentro es un develamiento de intereses y de encuentros en medio de la pluralidad.

En síntesis el objeto de intervención ha sido una de las categorías menos desarrolladas dentro del trabajo social, la cual a su vez ha sido tema de debate por muchos profesionales, esto visto en la década de los 70, 80 y 90 donde el tema de la Reconceptualización era relativamente reciente, ¿pero qué pasó después de esos años?, pareciera por la revisión bibliográfica que en su gran mayoría las y los trabajadores sociales dejaron de preocuparse, por lo menos en Iberoamérica y en las dos últimas décadas, por establecer cuestionamientos entorno a esta categoría, entrando así en lo que podría denominarse como un conformismo teórico, o lo que autores como Casa ( 2016) ubican como una pérdida de identidad por la profesión y disciplina; en el sentido que si no nos preguntamos por el objeto, no le cuestionamos e interrogamos a la luz de los contextos particulares, estaremos generando acciones estériles, con fronteras borrosas, sincrónicas e incluso carentes de sentido disciplinar.

El no preguntarse por la construcción histórica del objeto de intervención en trabajo social tiende a convertir a los nuevos profesionales, en sujetos a-históricos, que conocen de temáticas pero desconocen la configuración epistémica de la profesión. En ese sentido, encontramos en la revisión documental, por ejemplo, colegas que seguían utilizando de manera anacrónica e incluso descontextualizada, definiciones de autores que se encontraban inmersos en distintos contextos históricos y con problemáticas diferentes a las actuales o a las de su contexto particular. De acuerdo a lo 
Juliana Amaya Paulo, Carmen Melissa González Flórez, Estefani Gulloso Hernández, Angélica María Ramírez Martelo, Natalia Paola Torres López

anterior es necesario que desde nuestra disciplina y profesión replanteemos sobre la teorización de las diferentes categorías alrededor del Trabajo Social, y de esta manera inmiscuirnos en el debate como una característica propia de nuestra profesión-disciplina.

\section{REFERENCIAS BIBLIOGRÁFICAS}

Alayón, Norbeto \& Molina, Ma Lorena (2004). Acerca del Movimiento de Reconceptualización. Revista Prospectiva, Universidad del Valle. (10) 10.

Casá, Evangelina (2016). La producción de conocimiento en Trabajo Social: reflexiones desde el caso argentino. Datos de Trabajo Social- Revista de Trabajo Social y Acción Social. 54.

Calvo, Valentín (2010). El objeto de estudio en Trabajo Social: una visión desde el psicoanálisis, la ley y la estructura. Portularia. Huelva España.

De Martino, Mónica \& Espasandín, Cecilia (2003). Notas teóricas sobre prácticas profesionales. Revista de trabajo social Plaza Pública. 9.

De Lozano, Edelmira (2008). Objeto y modo de actuación del trabajador social. Boletín Electrónico Surá N 154. Escuela de Trabajo Social - Universidad de Costa Rica.

Del Valle, Alejandro \& Ramella, María (2009). De Trabajo Social y Ciencias Sociales: de la Reconceptualización al método crítico. Un viaje de vida. Revista trabajo social: universidad de Antioquia, 9.

Fernández, Josefina; Parra, Belén \& Torralba, Rosello (2012). Trabajo social con familias y resiliencia familia: análisis de la relación entre ambos conceptos. Atlántida: Revista Canaria de Ciencias Sociales, 14.

Galeana De La O, Silvia \& Tello, Nelia (2010). Fragilidad y debilidad del discurso en trabajo social: ausencia de la construcción desde la intervención social. Revista de Trabajo Social UNAM, 1.

González, Alicia (2015). Acerca de la Intervención. Revista Rumbos TS, 11. Universidad central de Chile. Chile.

González, Alicia (2014). El mito del "Objeto" en Trabajo Social. Revista Rumbos TS, 10. Universidad central de Chile. Chile.

Martínez, María \& Torrecilla, Agustina (2015). El objeto de intervención del trabajo social y su construcción a lo largo de la historia. Documentos de trabajo social: Revista de trabajo y acción social. 56.

Martínez, Laura (2015). Tiempos de mariposas y ruiseñores: una reflexión sobre el trabajo social en la reparación a víctimas del conflicto armado. Revista Palobra, 15. 
Micolta, Amparo; Escobar, María \& Betancourt, Lady (2013). La investigación y la intervención con familias. PROSPECTIVA, Colombia.

Parra, Gustavo (2007). El objeto y el Trabajo Social. Algunas aproximaciones a la problemática del objeto en el Trabajo Social. Boletín electrónico de Sura N 419. Escuela de Trabajo Social Universidad de Costa Rica.

Pérez, Alexander (2009). Re-pensar la Sistematización y la Investigación Evaluativa en la Intervención del Trabajo Social, como Pilares para la Producción de Conocimiento. Revista Palobra, 10(10).

Quintanilla, Oscar (2013). Factores sociohistóricos que configuran los sentidos y significados de la intervención social. Revista de Trabajo Social de la UNAL, 15. Universidad Nacional. Colombia.

Rivera, Daris (2014). Una mirada crítica sobre el objeto de estudio y el objeto de intervención en Trabajo Social. Boletín Electrónico Surá N 214. Escuela de Trabajo Social - Universidad de Costa Rica.

Ritzer, George (1993). Teoría Sociológica Contemporánea. Editorial Mcgraw-Hill, primera edición, España.

Sesma, Esther \& Girela, Blanca (2013). Trabajo Social Comunitario y construcción de paz, DTS (Documentos de Trabajo Social). №52. Universidad Granada. España.

Tello, Nelia (2010). Ires y venires de la intervención de Trabajo Social. Revista de Trabajo Social UNAM. Ciudad de México.

Tejada, Johanna; Rojas, Ginna \& Sierra, Angie (2013). Lecciones aprendidas del movimiento social feminista para la reflexión de la intervención en Trabajo Social. $\mathrm{N}^{\circ} 18$. PROSPECTIVA. Colombia.

Zurita, Ronald (2012). Propuesta de Objeto e Identidad profesional y una visión sobre Disciplina: Pensar, repensar y seguir pensando al Trabajo Social. Margen, 65. Universidad de Concepción. Chile. 

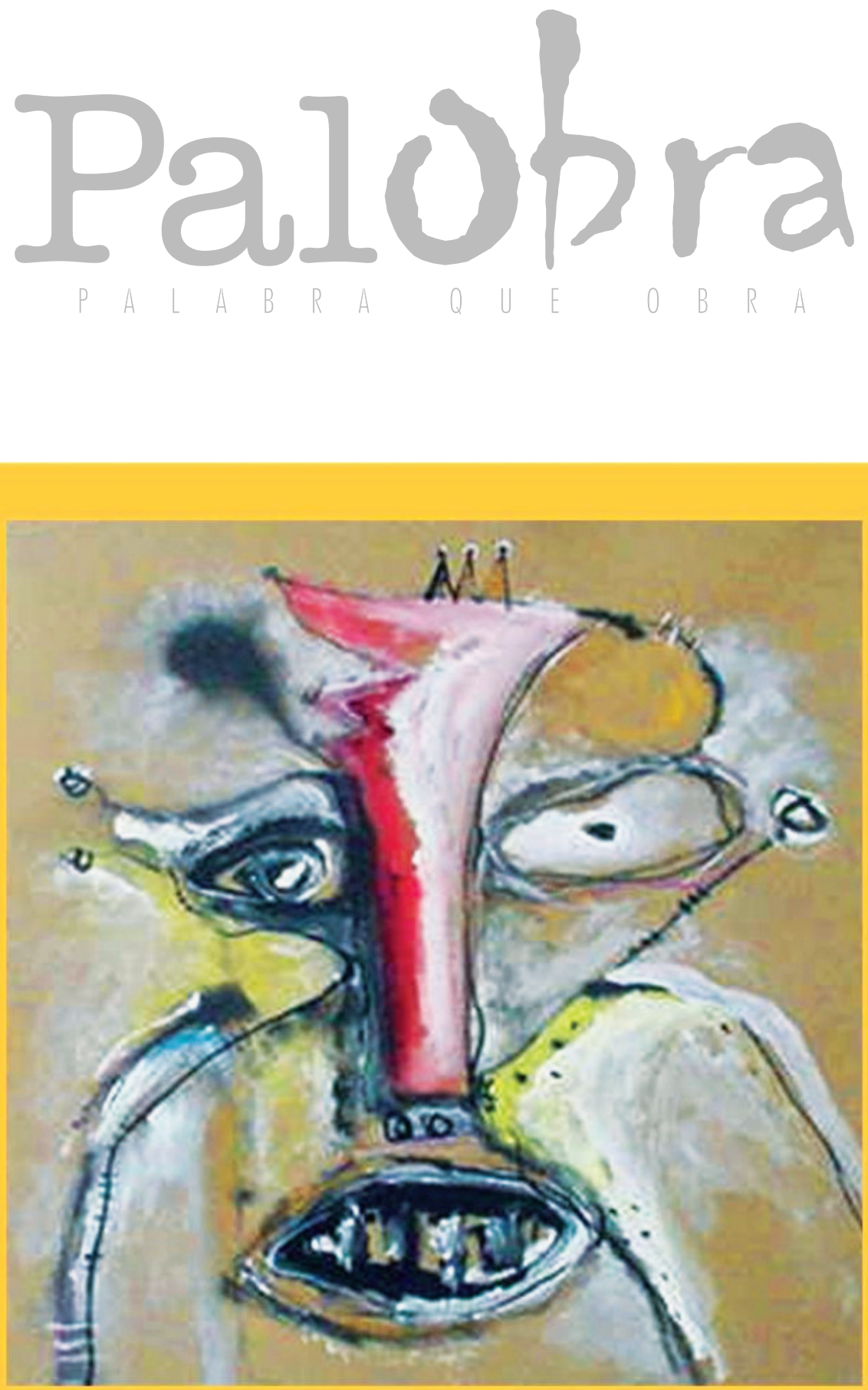

Obra pictórica de la serie "Rostros" de Eduardo Potrillé 